\title{
Surgical treatment of children Graves' disease with huge goiter- a case report and literature review
}

\author{
Yao Li ${ }^{1 \#}$, Xiang Cui ${ }^{2 \#}$, Yongjun Yang ${ }^{3}$, Yan Liang ${ }^{2}$, Fan Chai ${ }^{1}$, Yi-Ceng Sun ${ }^{1}$, Cong Shao ${ }^{1}$, Hongbiao Mo ${ }^{1}$, \\ Supeng Yin ${ }^{1}$, Zeyu Yang ${ }^{1}$, Fan Zhang ${ }^{1}$ \\ ${ }^{1}$ Breast and Thyroid Surgical Department, Chongqing General Hospital, University of Chinese Academy of Sciences, Chongqing 400000, China; \\ ${ }^{2}$ Breast and Thyroid Surgical Department, Southwest Hospital, Third Military Medical University (Army Medical University), Chongqing 400000, \\ China; ${ }^{3}$ Hepatobiliary and Pancreatic Surgery Department, Chongqing General Hospital, University of Chinese Academy of Sciences, Chongqing \\ 400000, China \\ "These authors contributed equally to this work. \\ Correspondence to: Fan Zhang. Breast and Thyroid Surgical Department, Chongqing General Hospital, University of Chinese Academy of Sciences, \\ Avenue of Stars 118, Liangjiang New District, Chongqing 400000, China. Email: zhangfan316@163.com.
}

\begin{abstract}
Graves' disease is the most common cause of hyperthyroidism in children. The surgery treatment for children Graves' disease with huge goiter is high risk and controversial. A 14-year-old girl suffered Graves' disease with huge goiter and failed to the antithyroid drug therapy for nearly 4 years was surgically treated with total thyroidectomy. The excised thyroid weighed $449.1 \mathrm{~g}$ and heavier than any excised children goiter reported so far. After operation, the patient's symptoms of Graves' disease were significantly improved without any complication, including normal basal metabolic rate, relieved exophthalmia and euthyroidism. So, a children Graves' disease with huge goiter was cured by total thyroidectomy, suggesting that a total/ near-total thyroidectomy is a good option for children Graves' disease with huge goiter.
\end{abstract}

Keywordsı Children; Graves' disease; goiter; surgery; case report

Submitted Dec 18, 2019. Accepted for publication Feb 18, 2020.

doi: $10.21037 /$ gs.2020.02.19

View this article at: http://dx.doi.org/10.21037/gs.2020.02.19

\section{Introduction}

Graves' disease (GD) is a systemic autoimmune disorder caused by the stimulatory autoantibodies $(\mathrm{Ab})$, which activates the thyroid-stimulating hormone receptor (TSH-R) leading to thyroid hyperplasia and unregulated thyroid hormone production and secretion (1). The patients of GD commonly manifest as symptoms of hyperthyroidism, such as weight loss, fatigue, heat intolerance, tremor, palpitations, goiter, bilateral proptosis, localized dermopathy and acropachy (2). GD is the most common cause of hyperthyroidism in both of children and adults (3), but the incidence rate of children is lower than adult. In children, girls had a significantly higher incidence than boys in the 10- to 14-year age group (4). The treatment strategies for GD include decreasing thyroid hormone synthesis with antithyroid drug (ATD), reducing the amount of thyroid tissue with radioactive iodine (RAI) and thyroidectomy (5). ATD is the firstline treatment strategy for the children's GD due to the obviously advantages with outpatient therapy, low risk of hypothyroidism, no radiation hazard or surgical risk (6). However, ATD only has a remission rate of $40-50 \%$ and a high recurrence rate with many side effects, such as rash, urticaria, arthralgia, fever, nausea, and abnormalities of taste or smell (7). The RAI and thyroidectomy are necessary when ATD failed. Despite of RAI has been used as a normal outpatient procedure with low cost since 1941, it has potential radiation hazards, radiation thyroiditis and hypothyroidism, even an increasing risk of cancer or genetic damage for children (8). In addition, the efficacy of RAI is decreasing with the increasing of goiter size. Thyroidectomy is the least choice for treatment of GD failed to ATD and RAI regimens, although it has many disadvantages, such 

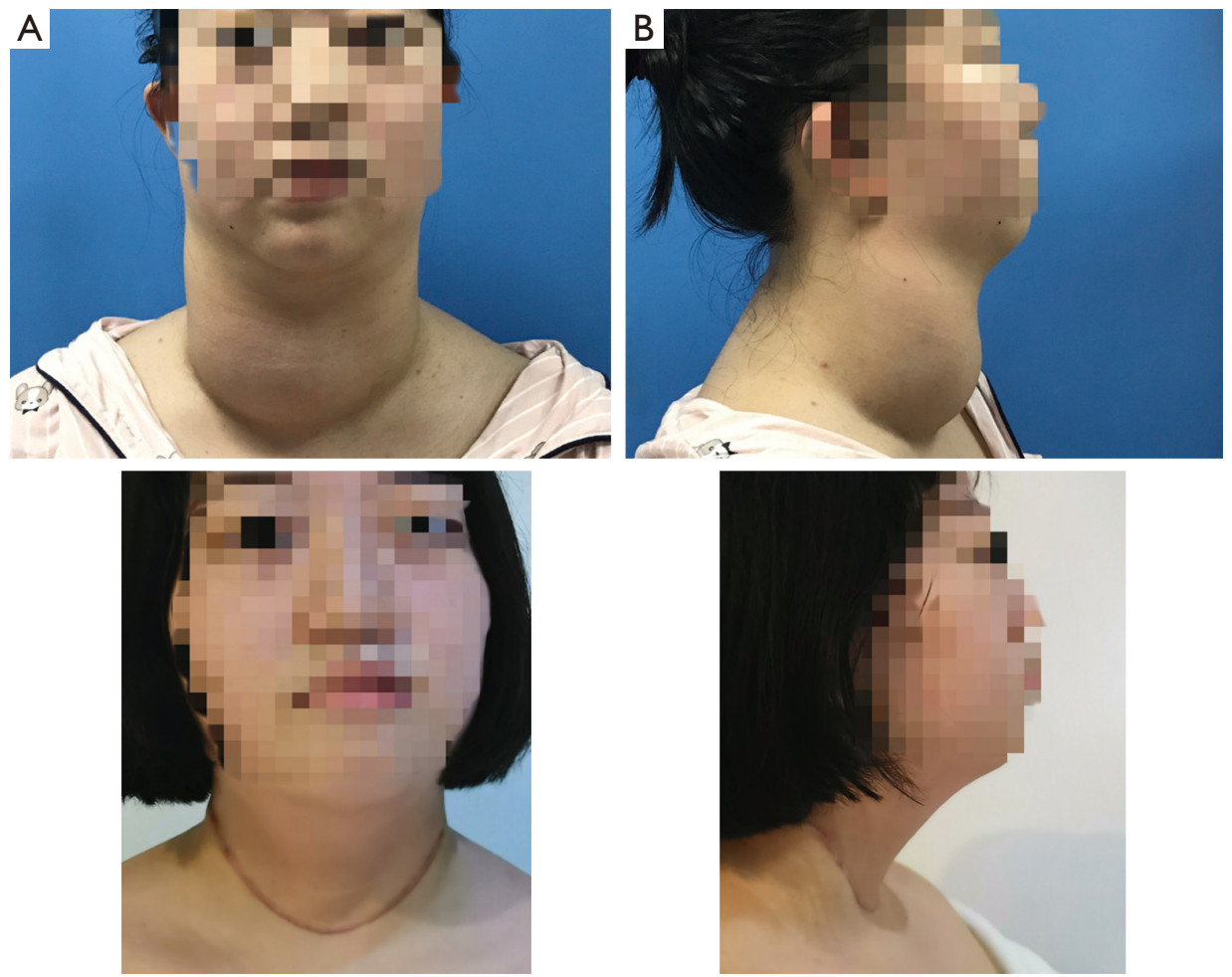

Figure 1 Huge goiter of the patient. A huge goiter in front of the patient's neck. (A) Front view; (B) side view (up: preoperative; bottom: postoperative).

as hypothyroidism, hypoparathyroidism and vocal cord dysfunction, tracheal collapse and softening, the permanent scar and long-term Levothyroxine replacement therapy (9). Thyroidectomy is also considered as first-line option for adult's GD in recent years with rapid euthyroidism, extremely rare recurrence, no radiation hazard, definitive histologic results and rapid relief of symptoms (10). It is well known that euthyroidism is very important for the growing and development of children. So thyroidectomy in children is usually performed with discretion. In the present report, the patient had severe GD with a huge goiter accompanied trachea compression. Therefore, we performed a total thyroidectomy for the patient. After the surgical treatment, the patient recovered without complications. The excised thyroid weighed $449.1 \mathrm{~g}$, which is heavier than any excised children goiter reported so far. In addition, we reviewed the surgical treatment of children GD.

\section{Case presentation}

A 14-year-old girl was diagnosed as hyperthyroidism in
April 2015, and treated with oral methimazole (MMI) combined Levothyroxine (specific dose unknown). The free triiodothyronine (FT3), free thyroxine (FT4) and thyroidstimulating hormone (TSH) returned to normal after the treatment for 5 months. However, the patient found that her thyroid begun to enlarge in March 2018, implying her hyperthyroidism has recurred. Treated with the original treatment regimen of MMI combined levothyroxine for about one year, her thyroid did not shrink, instead of gradual enlargement. So the patient visited our hospital in March 2019. GD was diagnosed based on the following observations: a huge mass in front of the neck (Figure $1 A$ up, Figure $1 B \mathrm{up}$ ), bilateral exophthalmia (Figure 2). FT3 $21.64 \mathrm{pmol} / \mathrm{L}$ (normal range, 3.1-6.8 pmol/L), FT4 $4.82 \mathrm{pmol} / \mathrm{L}$ (normal range, $12-22 \mathrm{pmol} / \mathrm{L}$ ), TSH $<0.005 \mathrm{uIU} / \mathrm{mL}$ (normal range, 0.27-4.2 uIU/mL). Ultrasound scan showed diffuse and homogeneous enlargement of the thyroid gland (Figure 3). The trachea was significantly compressed by the enlarged thyroid and the narrowest part was only $5 \mathrm{~mm}$ in CT image (Figure 4). Fortunately, the patient had no dyspnea and hoarseness. Also, her other symptoms of hyperthyroidism 

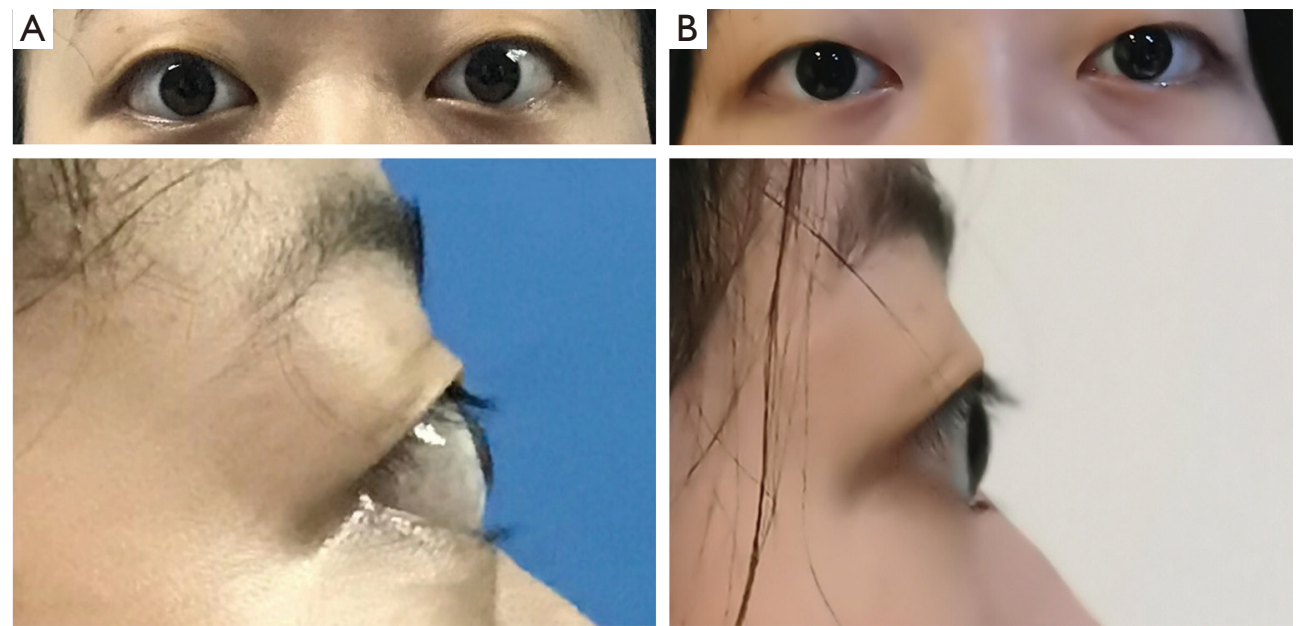

Figure 2 The exophthalmia of the patient. The patient manifests a significant bilateral exophthalmia before surgery and marked recovery after operation. (A) Preoperative; (B) postoperative (up: front view; bottom: side view).
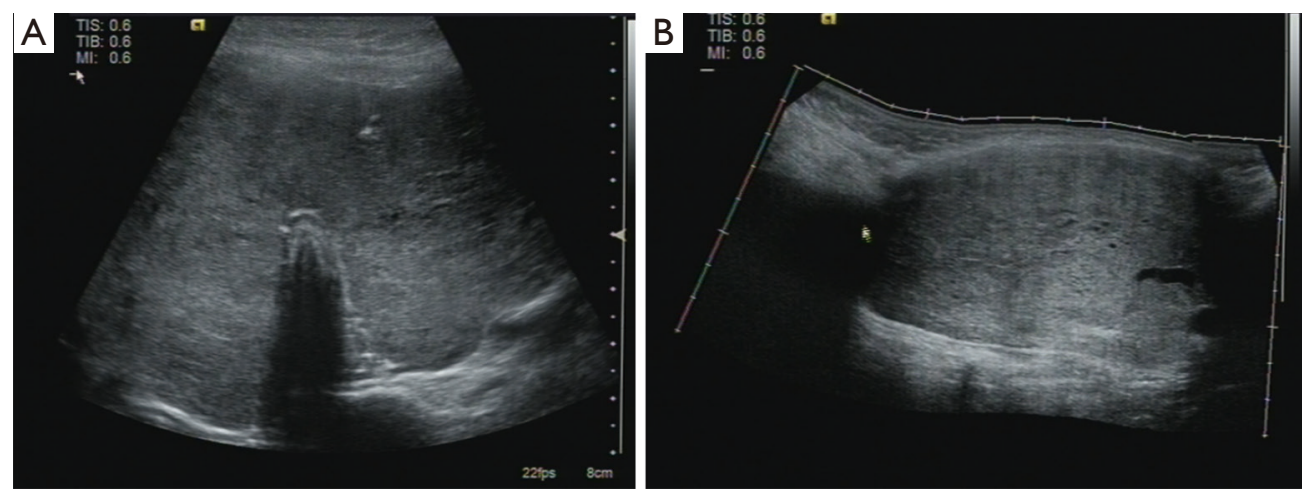

Figure 3 Ultrasound scan images of the goiter. Ultrasound scan displays a diffuse and homogeneous enlargement of the thyroid glands. (A) Transverse section (B) longitudinal section.

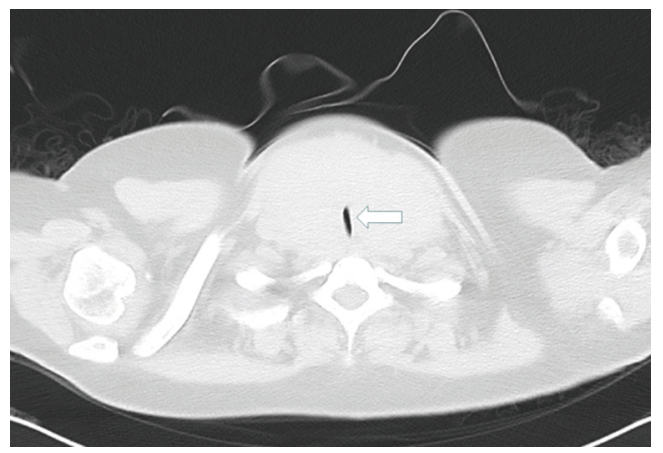

Figure 4 CT image of the goiter. CT image shows markedly enlarged thyroid and a significantly compressed trachea with about $5 \mathrm{~mm}$ at the narrowest part. were unconspicuous due to MMI treatment, such as excessive sweating, overeating, fatigue, irritability, poor concentration, nervousness, palpitations and tremors.

On April 17, 2019, a total thyroidectomy was performed for the patient when her basal metabolic rate (BMR) was regulated less than $20 \%$ and vascularity of the gland was decreased by compound iodine. During the operation, the thyroid gland was completely excised (Figure $5 A$ ) with the left upper parathyroid gland in situ retained. The size of excised thyroid was $12 \mathrm{~cm}$ in superoinferior diameter (Figure $5 B$ ), $5 \mathrm{~cm}$ in anteroposterior diameter, $15 \mathrm{~cm}$ in the transverse diameter with $449.1 \mathrm{~g}$ in weight (Figure 5C). The pathological diagnosis ultimately confirmed the Graves' 

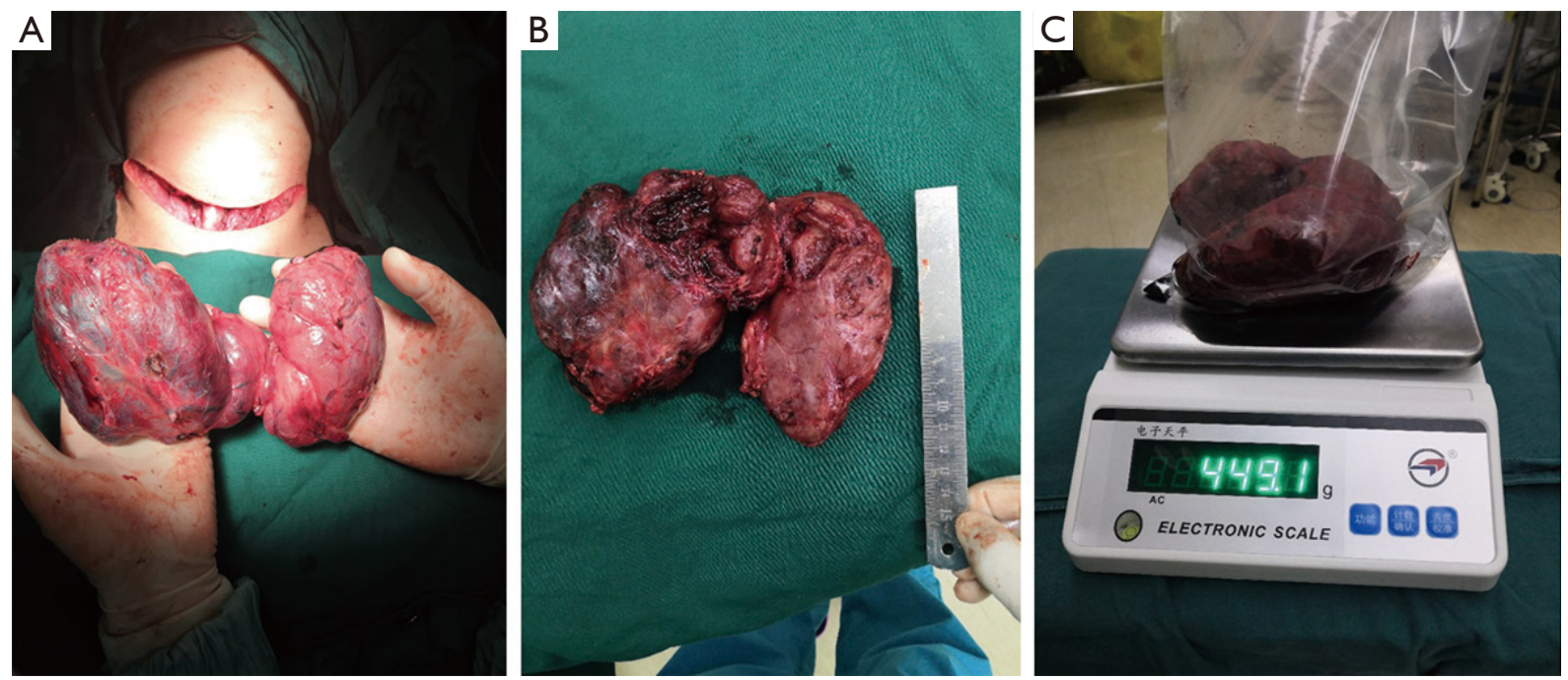

Figure 5 The excised enlarged thyroid glands of the patient. (A) The enlarged thyroid glands of the patient are completely excised. (B) The maximal superoinferior diameter of excised thyroid glands is $12 \mathrm{~cm}$. (C) The excised thyroid glands weight $449.1 \mathrm{~g}$.
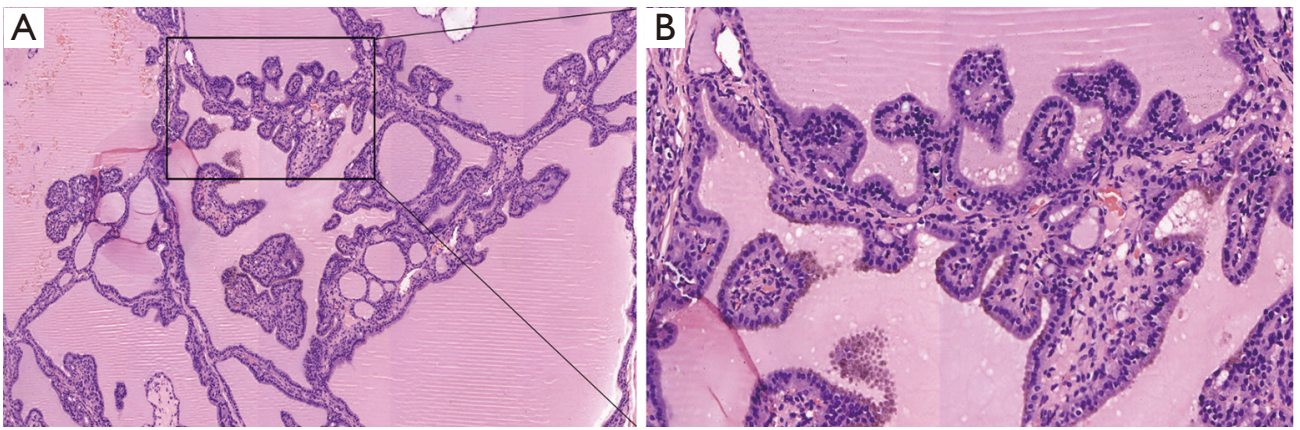

Figure 6 The pathologic image of excised goiter. Pathological image of the excised thyroid glands shows a Graves' disease feature. Magnification: 10 times (left), 20 times (right).

$\begin{aligned} & \text { The patient was diagnosed } \\ & \text { with hyperthyroidism }\end{aligned}$
$\begin{aligned} & \text { The patient's thyroid } \\ & \text { begun to enlarge }\end{aligned}$

Figure 7 The timeline figure of historical and current information.

disease (Figure 6). After surgery, the patient recurred quickly under the Levothyroxine replacement therapy. Follow up showed the patient was in good condition at the fifth month after surgery treatment (Figure $1 A$ bottom, Figure $1 B$ bottom, Figure $2 B$ up and bottom). The treatment timeline of the patient was summarized as Figure 7.

\section{Discussion}

The treatment for children GD is aimed to ensure the appropriate metabolism, growth, and neurocognitive development for the children by correcting abnormal thyroid function (11). Usually, a children GD patient is firstly experienced ATD to reduce thyroid hormone 
synthesis. When a patient is failure to ATD (12), the RAI treatment is performed to reduce the amount of thyroid tissue. Recent years, surgical treatment as a radical therapy for children GD has been recognized by more people and has gained more applications, especially for the patients who are failure to ATD or RAI, or have a huge goiter (13). Sherman et al. reported in 2006 that 69 of 76 children with GD chose operative therapy because of failure of medical therapy or adverse drug reactions (14). Elfenbein $e t a l$. found that all of the 31 pediatric patients with hyperthyroidism chose surgery as the cure treatment not RAI when ATD failed in their report, and the thyroidectomy-specific complications in children were acceptably when performed at high-volume centers (15). A case report from Japan showed that 4 of the 5 ATD failed patients had surgery (16). A retrospective study from the Journal of Pediatric Surgery showed that 124 children with GD chose surgery treatment in a single center between 2009 and 2017 (17). Overall, the thyroidectomy for pediatric GD is a definitive and highly effective treatment when performed by experienced thyroid surgeon. However, it's controversial that the subtotal thyroidectomy and total/ near-total thyroidectomy for surgery treatment of children GD (15). Subtotal thyroidectomy was considered minimize the risk of decreased quality of life due to complications, such as permanent hypoparathyroidism and vocal cord dysfunction, but it has an $8 \%$ chance of persistence or recurrence of hyperthyroidism at 5 years (18). Total/neartotal thyroidectomy has a nearly $0 \%$ risk of recurrence, and it's reported that there was no increase of postoperative complications in the total/near-total thyroidectomy group compare to the subtotal thyroidectomy group (19). In the present case report, we performed a total thyroidectomy for a children GD patient failed to ATD and accompanied a huge goiter, and achieved an ideal effect. In order to reduce the rate of complications related to thyroidectomy of GD, the following strategies should be considered.

\section{Preoperative}

Firstly, surgery should be performed after controlling the basal metabolic rate (BMR) less than 20\% and the heart rate below 90 by administrating MMI (20 mg, twice daily) and propranolol (10 mg, twice daily). In addition, the vascularity of the gland should be decreased by compound iodine (from 5 drops a time, three times a day, plus 1 drop each time, up to 16 drops a time, three times a day until the day of surgery) in the 2 weeks preceding surgery. Neck-enhanced
CT should be performed preoperatively to obtain the local anatomical structure of the surgery area.

\section{Intraoperative}

To ensure safety, the general anesthesia should be chosen. The most suitable tracheal tube was selected according to the compression of trachea, and the intubation should be performed in the case of waking. During operation, the exquisite surgical procedures are needed to avoid excessive bleeding, even the use of compound iodine before surgery to decrease the vascularity of the gland. Especially, when thyroid weigh more than $200 \mathrm{~g}$, the preparation for blood transfusion should be considered for safe (20). Furthermore, it is difficult to protect the recurrent laryngeal nerves because the nerves usually leaved the normal anatomical location in the GD patient with huge goiter and the intraoperative nerve monitoring is impossible due to the compressed trachea. In addition, parathyroid should be preserved in situ as far as possible, and the autotransplantation should be performed in case it is removed. So a high-volume surgeon is required for the thyroidectomy of GD with huge goiter to decrease the risk of complications. And the corresponding author in our article performed more than 800 thyroidectomies per year. This may be an important factor for the success of surgical treatment of huge children goiter.

\section{Postoperative}

The special complications of thyroidectomy for the GD with huge goiter include tracheal collapse and softening. So a preventive tracheal suspension would be performed in operation based on the rigidity of trachea. For patients' safety, a tracheotomy package is routinely prepared at the bedside in order to perform urgently tracheotomy once dyspnea is occurred (21). Another special complication of thyroidectomy for the GD with huge goiter, thyroid storm, should be also alerted despite the rare. The thyroid storm is a life-threatening endocrine state characterized by myasthenia, cardiovascular symptoms, in particular tachycardia, as well as hyperthermia and central nervous system dysfunction (22). Once it occurred, the patient should be given the critical care measures therapy, antithyroid drug treatment, rest, sedation, fluid and electrolyte replacement, cardio-supportive therapy, oxygen therapy, antibiotics and cooling. Last but not least, the patient of children GD must receive a long-term 
Levothyroxine replacement therapy, which has been proved to be effective and rare side effects.

In summary, this report has three strengths: (I) a GD patient failed to ATD and accompanied a huge goiter is cured by total thyroidectomy, suggesting a total/neartotal thyroidectomy might be a good option for children Graves' disease with huge goiter; (II) the excised thyroid is heavier than any excised children goiter reported so far; (III) the surgical treatment of children GD are reviewed, including preoperative, intraoperative and postoperative strategies. However, four limitations for this case should be noticed: (I) the patient originally received ATD treatment for hyperthyroidism for 5 months in April 2015, but recurred with the enlargement of thyroid in March 2018, implying the original ATD treatment is inadequate. (II) Although a total thyroidectomy would avoid recurrence of hyperthyroidism, the patient needed lifelong replacement therapy with levothyroxine. (III) A sufficient preoperative preparation and skilled surgical techniques are needed for the surgery to avoid the thyroid storm and complications. (IV) the experience of one special case may not apply to all patients, thus further studies are required.

\section{Acknowledgments}

Special thanks to professor Youhong-Cui for modifying of the manuscript.

Funding: This work is supported by the Science and Technology Innovation Plan of Southwest Hospital (SWH2016YSCXZD-10).

\section{Footnote}

Conflicts of Interest: All authors have completed the ICMJE uniform disclosure form (available at http://dx.doi. org/10.21037/gs.2020.02.19). The authors have no conflicts of interest to declare.

Ethical Statement: The authors are accountable for all aspects of the work in ensuring that questions related to the accuracy or integrity of any part of the work are appropriately investigated and resolved. Written informed consent was obtained from the patient for publication of this manuscript and any accompanying images.

Open Access Statement: This is an Open Access article distributed in accordance with the Creative Commons Attribution-NonCommercial-NoDerivs 4.0 International
License (CC BY-NC-ND 4.0), which permits the noncommercial replication and distribution of the article with the strict proviso that no changes or edits are made and the original work is properly cited (including links to both the formal publication through the relevant DOI and the license). See: https://creativecommons.org/licenses/by-nc-nd/4.0/.

\section{References}

1. Teniente-Serra A, Soldevila B, Quirant-Sanchez B, et al. Distinct pattern of peripheral lymphocyte subsets in Graves' disease with persistency of anti-TSHR autoantibodies. Autoimmunity 2019;52:220-7.

2. Ehlers $M$, Schott $M$, Allelein S. Graves' disease in clinical perspective. Front Biosci (Landmark Ed) 2019;24:35-47.

3. Smith TJ, Hegedus L. Graves' Disease. N Engl J Med 2016;375:1552-65.

4. Srinivasan S, Misra M. Hyperthyroidism in children. Pediatr Rev 2015;36:239-48.

5. Bartalena, Luigi. Diagnosis and management of Graves disease: a global overview. Nat Rev Endocrinol 2013;9:724-34.

6. Okawa ER, Grant FD, Smith JR. Pediatric Graves' disease: decisions regarding therapy. Curr Opin Pediatr 2015;27:442-7.

7. Cooper DS. Antithyroid drugs. N Engl J Med 2005;352:905-17.

8. Rivkees SA, Dinauer C. An optimal treatment for pediatric Graves' disease is radioiodine. J Clin Endocrinol Metab 2007;92:797-800.

9. Smithson M, Asban A, Miller J, et al. Considerations for Thyroidectomy as Treatment for Graves Disease. Clin Med Insights Endocrinol Diabetes 2019;12:1179551419844523.

10. Snyder S, Govednik C, Lairmore T, et al. Total thyroidectomy as primary definitive treatment for Graves' hyperthyroidism. Am Surg 2013;79:1283-8.

11. Jennifer W, Taback SP, Sellers EAC, et al. Graves' disease in children. Best Pract Res Clin Endocrinol Metab 2014;28:233-43.

12. Rivkees SA. Controversies in the management of Graves' disease in children. J Endocrinol Invest 2016;39:1247-57.

13. Lee HS, Jin SH. The treatment of Graves' disease in children and adolescents. Ann Pediatr Endocrinol Metab 2014;19:122-6.

14. Sherman J, Thompson GB, Lteif A, et al. Surgical management of Graves disease in childhood and adolescence: an institutional experience. Surgery 2006;140:1056-61; discussion 1061-2. 
15. Elfenbein DM, Katz M, Schneider DF, et al. Thyroidectomy for Graves' disease in children: Indications and complications. J Pediatr Surg 2016;51:1680-3.

16. Tomari K, Goto M, Shimada A, et al. Five cases of childhood-onset Graves' disease treated with either surgery or radio-iodine therapy. Clin Pediatr Endocrinol 2017;26:265-9.

17. Baumgarten HD, Bauer AJ, Isaza A, et al. Surgical management of pediatric thyroid disease: Complication rates after thyroidectomy at the Children's Hospital of Philadelphia high-volume Pediatric Thyroid Center. J Pediatr Surg 2019;54:1969-75.

18. Sung TY, Lee YM, Yoon JH, et al. Long-Term Effect of Surgery in Graves' Disease: 20 Years Experience in a

Cite this article as: Li Y, Cui X, Yang Y, Liang Y, Chai F, Sun YC, Shao C, Mo H, Yin S, Yang Z, Zhang F. Surgical treatment of children Graves' disease with huge goiter-a case report and literature review. Gland Surg 2020;9(2):467-473. doi: 10.21037/ gs.2020.02.19
Single Institution. Int J Endocrinol 2015;2015:542641.

19. Wilhelm SM, Mchenry CR. Total Thyroidectomy Is Superior to Subtotal Thyroidectomy for Management of Graves' Disease in the United States. World J Surg 2010;34:1261-4.

20. Yamanouchi K, Minami S, Hayashida N, et al. Predictive factors for intraoperative excessive bleeding in Graves' disease. Asian J Surg 2015;38:1-5.

21. Kahaly GJ, Bartalena L, Hegedus L, et al. 2018 European Thyroid Association Guideline for the Management of Graves' Hyperthyroidism. Eur Thyroid J 2018;7:167-86.

22. Akamizu T. Thyroid Storm: A Japanese Perspective. Thyroid 2018;28:32-40. 